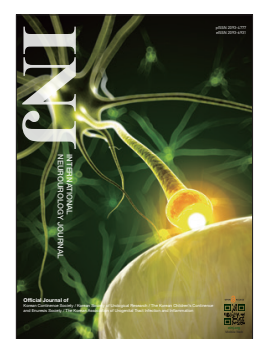

\title{
Detecting Bladder Biomarkers for Closed-Loop Neuromodulation: A Technological Review
}

\author{
Eunkyoung Park ${ }^{1}$, Jae-Woong Lee ${ }^{2}$, Minhee Kang ${ }^{1,2}$, Kyeongwon Cho ${ }^{1,2}$, Baek Hwan Cho ${ }^{1,2}$, Kyu-Sung Lee ${ }^{1,2,3}$ \\ ${ }^{1}$ Smart Healthcare \& Device Research Center, Samsung Medical Center, Sungkyunkwan University School of Medicine, Seoul, Korea \\ ${ }^{2}$ Department of Medical Device Management and Research, SAIHST, Sungkyunkwan University, Seoul, Korea \\ ${ }^{3}$ Department of Urology, Samsung Medical Center, Sungkyunkwan University School of Medicine, Seoul, Korea
}

Neuromodulation was introduced for patients with poor outcomes from the existing traditional treatment approaches. It is well-established as an alternative, novel treatment option for voiding dysfunction. The current system of neuromodulation uses an open-loop system that only delivers continuous stimulation without considering the patient's state changes. Though the conventional open-loop system has shown positive clinical results, it can cause problems such as decreased efficacy over time due to neural habituation, higher risk of tissue damage, and lower battery life. Therefore, there is a need for a closed-loop system to overcome the disadvantages of existing systems. The closed-loop neuromodulation includes a system to monitor and stimulate micturition reflex pathways from the lower urinary tract, as well as the central nervous system. In this paper, we reviewed the current technological status to measure biomarker for closed-loop neuromodulation systems for voiding dysfunction.

Keywords: Implantable neurostimulators; Voiding dysfunction; Urinary bladder diseases; Biomarkers

- Fund/Grant Support: This work was supported by Korea Health Technology R\&D Project through the Korea Health Industry Development Institute (KHIDI) funded by the Ministry of Health \& Welfare (HI14C3229), and the National Research Foundation of Korea (NRF) grant funded by the Korea government (MSIT) (NRF-2016R1C1B2010114).

- Conflict of Interest: No potential conflict of interest relevant to this article was reported.

\section{INTRODUCTION}

Urinary bladder is controlled by the central and peripheral nervous system to store and void urine. The voiding dysfunction may develop due to internal or external trauma, diseases, or injuries and is treated through various approaches of behavioral modification, pharmacotherapy, intradetrusor injections of onabotulinum toxin A (Botox, Allergan Inc., Irvine, CA, USA), or reconstructive surgery [1-6]. For patients with poor out- comes from the existing traditional treatment approaches, neuromodulation is well-established as an alternative, novel treatment option for voiding dysfunction [7-10].

Neuromodulation was introduced initially in the late 1870s to treat lower urinary tract dysfunction by directly applying electrical stimulation on the bladder using a transurethral electrode. Later, the scope of stimulation research transitioned away, from directly targeting the organ of interest to targeting the peripheral and sacral nerves. An implantable sacral elec-
Corresponding author: Kyu-Sung Lee (iD https://orcid.org/0000-0003-0891-2488 Department of Urology, Samsung Medical Center, Sungkyunkwan University School of Medicine, 81 Irwon-ro, Gangnam-gu, Seoul 06351, Korea E-mail: ksleedr@skku.edu / Tel: +82-2-3410-3554 / Fax: +82-2-3410-3027 (i) (2) This is an Open Access article distributed under the terms of the Creative Commons Attribution Non-Commercial License (http://creativecommons.org/licenses/by-nc/4.0/) which permits unrestricted non-commercial use, distribution, and reproduction in any medium, provided the original work is properly cited.

Co-corresponding author: Baek Hwan Cho (iD https://orcid.org/0000-0002-4106-9042 Smart Healthcare \& Device Research Center, Samsung Medical Center, 81 Irwon-ro, Gangnam-gu, Seoul 06351, Korea

E-mail: baekhwan.cho@samsung.com / Tel: +82-2-3410-0885 / Fax: +82-2-3410-2968

Submitted: November 6, 2018 / Accepted after revision: December 11, 2018 
trode, which the development began in the early 1980s by Tanagho et al. [11], provided a basis for the concept of contemporary sacral neuromodulation technology and the conventional device (Medtronic InterStim, Medtronic, Minneapolis, MN, USA) $[12,13]$. Additionally, around this time, McGuire et al. [14] discovered the inhibition of bladder overactivity by electrical stimulation of the tibial nerve. This became a basis for the concept of posterior tibial nerve stimulation (PTNS), which is widely used for the treatment of overactive bladder [15].

Such aforementioned neuromodulation systems can be categorized into either an open- or a closed-loop paradigm [16-18]. While the closed-loop system of neuromodulation uses a sensor that detects and records various symptom-related signals, the open-loop system does not use a sensor and stimulates continuously. Accordingly, the relevant parameters, such as duration, amplitude and frequency of applied electric stimulation, remain constant in the open-loop system. In the closed-loop system, on the other hand, the signals recorded or detected are considered as biomarkers, which are relevant to the physiology, pathology, biochemistry, state of disease and many others. Because physicians need to track and monitor the clinical status of a patient attentively and acquire the optimal set of stimulation parameters for treatment via a trial-and-error approach, the open-loop system cannot flexibly adjust the preset parameters, in accordance with the pathophysiological changes of the disorder in real time. On the other hand, the closed-loop system can analyze the measured biomarkers, and automatically and dynamically adjust the stimulation, all in accordance with the changing state of the disease and patient. Although the existing open-loop neuromodulation system continues to be a successful option for treatment, the closed-loop neuromodulation has profound potentials to treat and improve on voiding dysfunction in a more optimized sense.

Cardiac pacemaker was the first implantable electrical stimulation devices and a long-standing and successful history in the field of closed-loop neuromodulation [19,20]. In 1958, Lillehei and Bakken first introduced a battery-powered external pacemaker and reported it to be effective in 18 patients [21,22]. For 20 years, the pacemaker was designed in an open-loop manner to deliver constant electric pulses for treatment. In 1981, Rickards and Norman conceived the idea that pacing rate could be controlled by detection of the evoked response [22,23]. This became the foundation of today's closed-loop cardiac pacemaker. When technological advancements were made to enable the cardiac pacemaker to receive data from the heart in real time, new methods of treatment, such as implantable cardiac defibrillation, came into use as well [24]. Deep brain stimulation (DBS), which is now widely used in the patients with severe neurological disorders, also was recognized for its excellent clinical efficacy. Initially, DBS was used in the form of an openloop system; however, the open-loop form is consistently stimulated without consideration for the patient's dynamic status, and again requires the physician to manually adjust the stimulation parameters on a regular basis. Such could cause brain overstimulation or fewer benefits due to unnecessary stimulations. Therefore, many researchers have been investigating to advance in developing a closed-loop DBS system using various biomarkers, and are optimistic as to provide the maximized positive effects to the patients in need of the DBS treatment solution [25-29].

Though many research studies have demonstrated that the sacral neuromodulation is effective for treating voiding dysfunction, these studies applied preset stimulation protocols, without considering the dynamic nature of patient status or monitoring the physiological data in real time. No devices to date have applied the neuromodulation technology for voiding dysfunction by integrating the closed-loop feedback control $[13,30,31]$. PTNS is an alternative neuromodulation modality. It was investigated for its efficacy in different patient groups, and was shown to be approximately $40 \%-70 \%$ effective in treating the overactive bladder and urinary retention in particular [32-38]. The treatment using the current PTNS is typically administered once per week for 12 weeks, and is followed by the maintenance therapy if the patient shows any positive effect. This system, however, cannot function with the closed-loop control that can monitor the stimulation effect simultaneously and continuously, and has the disadvantage of relying solely on the physician's experience to achieve the optimized effect on a patient.

The closed-loop neuromodulation includes a system to monitor and stimulate micturition reflex pathways from the lower urinary tract, as well as the central nervous system. In addition, it is a fully automated system for which the patient does not need to provide a trigger. Such closed-loop feedback control aims to mimic the normal function of voiding by neuromodulation of the micturition reflex pathways. It is important to identify and measure suitable biomarkers to implement the closed-loop neuromodulation system. Therefore, in order to acquire the closed-loop control, it is technologically necessary to develop ways to monitor bladder biomarkers accordingly. In 
this paper, we will review the current technological status to measure the relevant biomarker for voiding dysfunction.

\section{WHAT ARE SOME CANDIDATE BIOMARKERS FOR A CLOSED-LOOP SYSTEM?}

Stimulation for controlling voiding function can be conducted either in the open- or closed-loop. The continuous stimulation in the open-loop system is effective in the inhibition of detrusor contractions and the recovery of bladder capacity. It should be noted, however, that the long-term, persistent stimulation can cause problems, such as decreased efficacy due to neural habituation, higher risk of tissue damage, and decreased battery life over time. Considering such issues, many researches underline the need to develop a novel approach of integrating the closedloop control. And, therefore, the search for and validation of appropriate feedback biomarkers are crucial.

The urinary bladder has 2 important functions: storage of urine and emptying. Disturbances of the storage function may result in lower urinary tract symptoms, such as urgency, frequency, and urge incontinence, as the components of the overactive bladder syndrome. The overactive bladder syndrome, which may be due to involuntary contractions of the smooth muscle of the bladder (detrusor) during the storage phase, is caused by increased pressure due to involuntary contraction. Therefore, the bladder pressure and contraction not only give physicians valuable insights into the overall health of a patient's bladder, but also important information in controlling micturition. Biomarkers that reliably reflect bladder events, such as bladder contraction or bladder pressure, can be one of candidates in implementing the closed-loop system to control micturition function (Table 1).

\section{HOW BIOMARKERS ARE MEASSURED FOR THE CLOSED-LOOP SYSTEM?}

The technology of measuring biomarkers has been developed because of the importance for the closed-loop feedback control system that can overcome the limits of conventional open-loop neuromodulation. In determining the methods of measuring biomarkers for the closed-loop system, it is important to focus on the feasibility of processing data, therapeutic purpose and considering pain. We will review the current technological status to measure these biomarkers for the closed-loop system.

\section{Electromyography}

The bladder contracts as its smooth muscle undergoes membrane depolarization to void urine. The electromyography (EMG) can be recorded from both the skin surface and subcutaneously, as well as intramuscularly. The signals simultaneously begin at the start of muscle contraction. The EMG from the bladder, the urethra, and the anal sphincter can be used to detect and quantify bladder events $[39,40]$. Though it had been difficult to acquire such signals accurately, due to low signal-to-

Table 1. Detection method of biomarkers for closed loop feedback neuromodulation system

\begin{tabular}{|c|c|c|}
\hline Biomarker & Group (yr) & Detection method \\
\hline \multirow[t]{5}{*}{ Bladder contractions } & Rutter et al. (2018) [56] & $\begin{array}{l}\text { Bladder contraction prediction model using sparse regression based on external urethral } \\
\text { sphincter electromyographic activity }\end{array}$ \\
\hline & Majerus et al. (2017) [55] & $\begin{array}{l}\text { Piezoelectric catheter-free pressure sensor to detect the bladder contraction in suburotheli- } \\
\text { al bladder location }\end{array}$ \\
\hline & Opisso et al. (2011) [53] & $\begin{array}{l}\text { Electromyography (EMG) was used to detect the bladder contractions in external urethral } \\
\text { sphincter }\end{array}$ \\
\hline & Horvath et al. (2010) [40] & $\begin{array}{l}\text { EMG algorithm provided to detection of bladder contractions in external anal sphincter for } \\
\text { conditional stimulation }\end{array}$ \\
\hline & Wenzel et al. (2005) [42] & Electroneurography activity for the bladder contraction detection in the pudendal nerve trunk \\
\hline \multirow[t]{5}{*}{ Bladder pressure } & Ross et al. (2018) [57] & Multiunit recordings to decode the bladder pressure from sacral-level dorsal root ganglia \\
\hline & Khurram et al. (2017) [61] & Suprapubic bladder catheters were implanted for saline infusion and the bladder pressure \\
\hline & Kim et al. (2017) [62] & Implantable sensor using the resistor ladder network to measure bladder volume \\
\hline & Majerus et al. (2017) [55] & Implantable wireless bladder pressure sensor for closed-loop neuromodulation system \\
\hline & Young et al. (2015) [60] & Implantable sensor for bladder pressure chronic monitoring by wireless recharging system \\
\hline
\end{tabular}


noise ratio in the past, the recent advancements in electrode and recording technology now allows data acquisition with high accuracy.

\section{Electroneurography}

Physiologically, the micturition activity involves coordination between the central, somatic and autonomic nervous systems. The electroneurography (ENG) recordings from the bladder afferent nerves is the suitable method to measuring and monitoring of the bladder volume. It is possible to measure the bladder volume recorded at specific spinal roots (i.e., dorsal root L6) that conduct signals from the bladder afferent nerves in animal models [41]. Furthermore, the bladder contraction can be detected by measuring the electrical activity of the pudendal nerve trunk $[39,42]$.

\section{Near-Infrared Spectroscopy}

Near-infrared spectroscopy (NIRS) is a relatively new noninvasive technique designed to monitor hemodynamics and oxygenation of the bladder. It is known to be the method of providing critical information to evaluate voiding dysfunction in patients. NIRS is unique in 2 ways: firstly, it is the only method or tool that is able to investigate the effect of the bladder's microcirculation on the maintenance of the bladder's contraction and relaxation; secondly, it is able to determine the influence of the detrusor muscle's hemodynamics and oxygenation on voiding function. Not only has NIRS been utilized in various studies of urological conditions for the voiding dysfunction, but it has been used to investigate testicular ischemic conditions, erectile dysfunction, and renal dysfunction as well [43-45]. In a number of human studies, NIRS can detect voiding changes with dynamic variation of oxygenated and deoxygenated hemoglo-
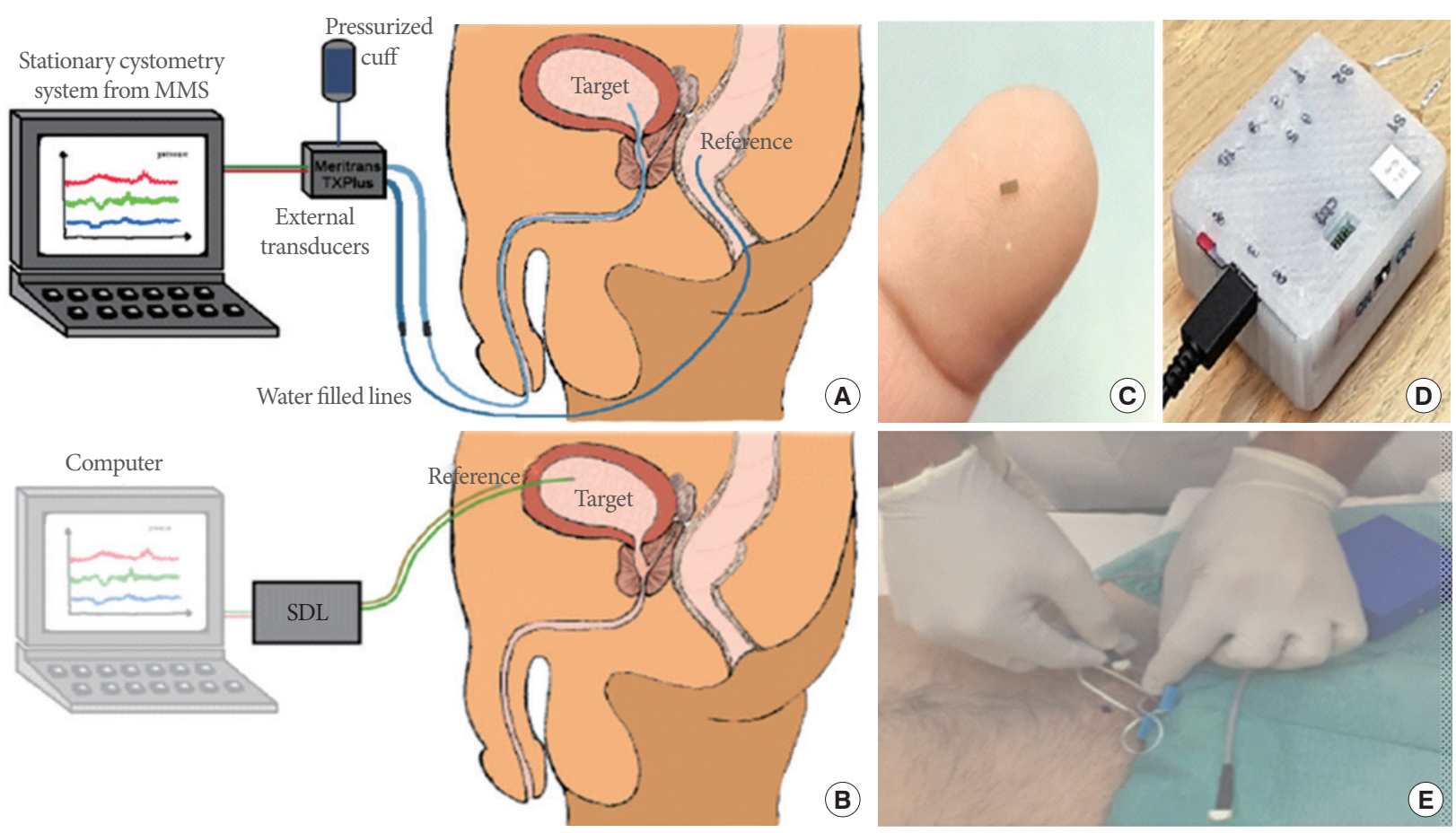

Fig. 1. A new cystometry system using a microelectromechanical system (MEMS)-based in-target bladder pressure sensor. (A) Bladder pressures are usually measured from water-filled lines that are connected to externally located transducers. The conventional method uses a catheter through the urethra into the bladder with a transducer in the distal end. This method is implemented with nonphysiological filling state and urethra obstructed by a catheter and produce artefacts by movements and measurement errors. (B) The MEMS-based system is developed in a suprapubic approach to resolve the several limitations of conventional method. It consists of a MEMS sensor (C), custom-made sensor data logger (SDL) (D), and reference sensor probe. This in-target organ pressure sensor is superior in measuring minute pressure pulses. This method is improving the quality of measurements and the option of long-term implantable devices. (E) The clinical trial. MMS, medical measurements systems. Reprinted from Clausen I, et al. Sensors (Basel) 2018 Jul 3;18(7), according to Open Access [51]. 
bin from the detrusor of the bladder [46]. Recently, a miniature wireless device was developed that uses light emitting diodes as a light source rather than lasers, and the device feasibility for wireless bladder monitoring was confirmed in the ambulant subjects [47-49]. In addition, Molavi et al. [50] developed a wireless, wearable sensor for measuring bladder fullness using NIRS, and confirmed its feasibility through both in vitro and in vivo tests.

\section{Implantable Bladder Sensors}

Bladder pressure is measured by direct insertion into the bladder through the urethra using a catheter-based sensor. However, this method is known to be inaccurate, because its measurement is affected by various factors of disturbance and resistance in the catheter line and the inertia of urine inside the bladder. In addition, after surgical intervention, this method can reduce the quality of life of patient and cause infection upon persistent use. The catheterization method is only suitable and recommended for short-term measurement. With the rapid development in microfabrication and machining, the miniaturized and sophisticated sensors are can be implanted into the patient's bladder for a long-term monitoring. Clausen et al. [51] adopted a MEMS (microelectromechanical system)-based system to detect the bladder pressure. This system was developed with a suprapubic approach to resolve the limitations of conventional catheterization method (Fig. 1). The implantable sensors can be used to acquire information for a long time. Therefore, since the status of the bladder can be transmitted in real time by the wireless catheter-free, the system can have the neuromodulation used for treating bladder dysfunction in a longer time [52].

\section{STUDIES OF THE TECHNOLOGY TO MEASURE BIOMARKERS}

\section{Bladder Contractions}

Though many researchers have attempted to identify bladder contractions with EMG measured from various areas, such as the detrusor, anal sphincter, and urethral sphincter, it is difficult to measure bladder EMG, for the recorded signal has very small amplitudes and the electrodes need to be implanted in the bladder. The patients with voiding dysfunction have either increased or decreased activities of the pelvic floor and anal sphincter when their bladder contracts. Changes in the anal sphincter EMG can be expected during the bladder contraction. A study by Horvath et al. [40] on the patients with spinal cord injury externally measured the anal sphincter EMG and used an adaptive threshold value as a trigger for simulation, and confirmed positive effects of the controlled stimulation from 2 patients in the study. In a different study by Opisso et al. [53], EMG from the external urethral sphincter was used to control the stimulation, and the patients with neurogenic detrusor overactivity had an $84 \%$ increase in bladder capacity. Accordingly, the detection of bladder contractions can be effectively utilized, if EMG can be measured in a chronically stable manner from the anal sphincter and the urethral sphincter muscles.

The pudendal nerve was used as a target to detect detrusor contractions via afferent nerve information. Wenzel and colleagues $[39,42]$ showed that a cuff-type electrode surrounding the pudendal nerve of a cat can be used to detect the bladder pressure of an average of $7 \mathrm{~cm} \mathrm{H}_{2} \mathrm{O}$ and bladder contraction in 1.2 seconds. They simultaneously used a urodynamic pressure value to verify the pudendal ENG and showed successful results in identifying certain bladder events. It, however, was challenging to find signals of a desired quality, since the pudendal nerve delivers various information, including that regarding the bladder, and because there was a low signal-to-noise ratio. In a human study using an electrode on the $\mathrm{S} 3$, it also was difficult to detect an accurate signal due to low signal-to-noise ratio, and to distinguish bladder pressure information from other signals while the nerve bundle received diverse information. Bruns et al. [54] conducted a study on the extraction of bladder filling by inserting microelectrode arrays in S1 and S2 of sacral dorsal root ganglia. They confirmed that the firing rate increases as the bladder fills to $11 \mathrm{~cm} \mathrm{H}_{2} \mathrm{O}$ in its pressure; furthermore, the firing rate decreases to nearly zero when the bladder is empty. This was possible to determine because of a higher signal-to-noise ratio due to the isolation of sensory afferents from motor fibers within both S1 and S2 dorsal root ganglia, unlike those in the S3.

Recently, promising results have been reported from studies using various implantable sensors to detect and monitor bladder contractions more conveniently. Majerus et al. [55] developed a novel piezoelectric catheter-free pressure sensor to detect bladder contractions. In feline and canine studies, the bladder contraction was measured from suburothelial and intraluminal locations, and was compared to the simultaneously measured pressure from a catheter in the bladder. They confirmed the correlation between the suburothelial pressure readings and the intravesical bladder pressure, and suggested the use of a single-channel submucosal pressure sensor to measure the bladder contractions reliably (Fig. 2). 

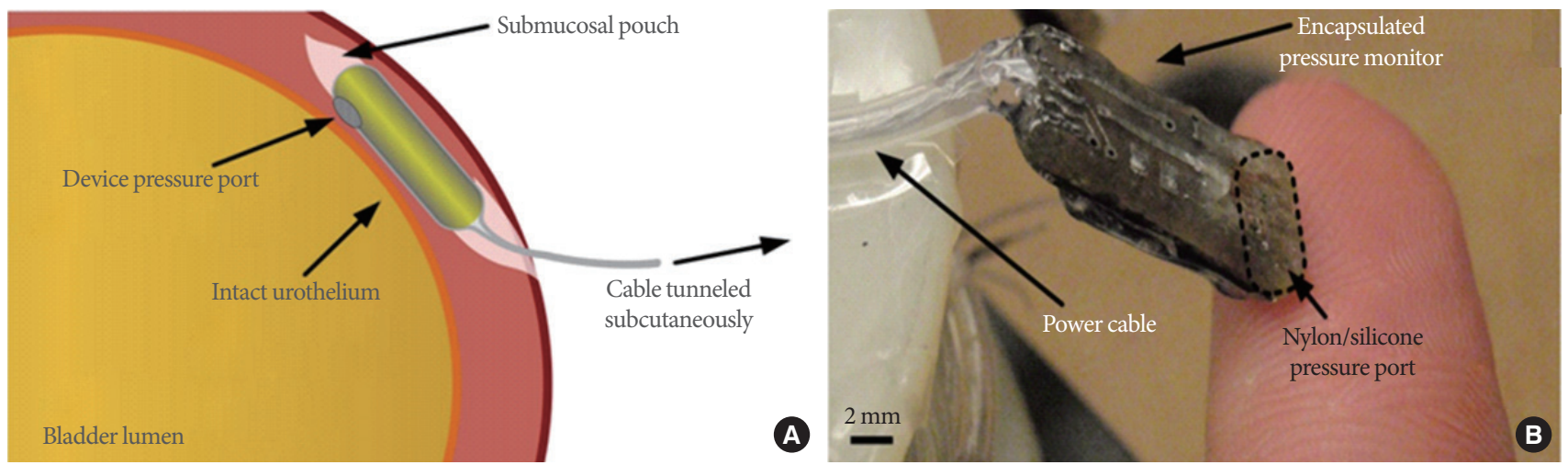

Fig. 2. Piezoelectric catheter-free pressure sensor to detect bladder contractions. The pressure measured in the submucosa correlates with the pressure of the bladder lumen pressures. The correlation coefficient is sufficient enough to measure bladder events such as detrusor contractions or abdominal compressions. Implantable sensor offers the opportunity for chronic monitoring. (A) Illustration of the wired pressure monitor of catheter-free, as implanted within the bladder wall. (B) Photograph of the fabricated implantable pressure monitor prototype. Reprinted from Majerus SJ, et al. PLoS One 2017;12:e0168375, according to Open Access [55].

Recently, not only is the bladder contraction being detectable, but also predictable methods are being studied. Rutter et al. [56] developed the method for predicting bladder contraction using a time-dependent spectrogram representation of external urethral sphincter EMG activity. This novel method enabled prediction of bladder contractions to the $91 \%$ specificity and $96 \%$ sensitivity. It is most suitable for implementing closedloop system to control micturition function.

\section{Bladder Pressure}

It is known that bladder fullness can be predicted by the pelvic nerve's reaction to changing bladder capacity, because the pelvic nerve afferent activity is a responsive action of nerve units to the bladder fullness. However, because the existing method of using pelvic nerve afferent activity requires laminectomy to dissect the L6 filaments, the sacral nerve is used instead of the pelvic nerve in many studies due to its relatively ease of access. While existing studies have attempted to estimate bladder pressure from single-unit recordings of the sacral dorsal root ganglia, Ross et al. [57] conducted a study attempting to decode bladder pressure from multiunit recordings of the dorsal root ganglia for seven anesthetized felines. They used feature selection methods and multivariate nonlinear time series models to analyze the detected signals, and suggested that the most accurate bladder pressure can be estimated from the nonlinear autoregressive moving average model. This study was the first attempt to decode bladder pressure using an algorithm based on signals detected from nerves.
Many researchers have reported that bladder pressure is more useful for extracting long-term bladder events. Therefore, they are now conducted to detect bladder pressure more directly using various sensors. In the mid-1980s, Brindley and Donaldson [58] adopted an implantable pressure sensor for the first time to stimulate pudendal nerves by muscular coat contraction every time bladder pressure increased. This was the first attempt at a device of closed-loop control using bladder pressure as a biomarker to treat severe urinary incontinence. The early stages of development for an implantable bladder pressure sensor were unsuccessful, due to problems with sensor drift, detachment, loss of accuracy over time, and infection. However, with the recent progress in biocompatible materials and micro-electro-mechanical system technologies, the resulting studies on bladder pressure detection are promising. Kim et al. [59] developed on a generic packaging method, based on encapsulating the sensor in medical grad polyurethane balloon, to decrease the baseline drift for implantable bladder sensors. Young et al. [60] developed the optimized implantable wireless battery recharging system. In a feline model, Khurram et al. [61] developed an integrated interface that can chronically monitor afferent activity of lower urinary tract through dorsal root ganglia during stimulation of a peripheral pathway. They were able to record the afferent activity of lower urinary tract continuously using penetrating microelectrode arrays inserted into pudendal nerves. Additionally, this system could yield potential uses in estimating bladder pressure and developing stimulation patterns and closed-loop control algorithms. Majerus et al. [55] developed a 
system that can monitor contraction as well as pressure wirelessly through chronic implantation into bladder muscles. This system is composed of an ultra-low-power circuit, a miniature pressure sensor, radio frequency antennas, and a rechargeable battery. Additionally, the system has undergone feasibility testing through large animal models, such as female Jersey calves. The researchers confirmed a correlation coefficient of 0.90 between bladder pressure acquired from their system and that from a vesical catheter, and the accuracy of pressure was related to the location of implantation. Kim et al. [62] recently tested an implantable sensor to monitor bladder volume that resolves existing problems like drift and device degradation. They developed and applied a 4-level resistor ladder in various lengths to monitor the gradual change of bladder volume. In addition, the adoption of a polypyrrole/agarose hydrogel composite enhanced this sensor in terms of biocompatibility and stability. It can be attached easily to the bladder, minimizing bladder tissue damage. Ex vivo experiments with pig bladders confirmed that resistor values of $1 \mathrm{k} \Omega, 0.5 \mathrm{k} \Omega$, and $0.75 \mathrm{k} \Omega$ correlated with bladder volumes of $80 \mathrm{~mL}, 130 \mathrm{~mL}$, and $220 \mathrm{~mL}$, respectively, and showed that an implantable sensor using a resistor ladder network can successfully measure bladder volume.

\section{CONCLUSIONS}

A better future for patients that need neuromodulation treatment is projected through recent technological advancements in neuromodulation for voiding dysfunction. In particular, neuromodulation using the closed-loop feedback control is expected to provide longer battery life, more efficient control of voiding dysfunction, and more effective voiding efficiency. The closed-loop systems can use biomarkers that help monitor the status of the bladder to maximize therapeutic benefits and minimize adverse effects. This is done by dynamically controlling stimulation type and pattern, which is fully and automatically programmable in accordance with the changing status of the patient. Furthermore, the closed-loop neuromodulation can be much more robust in action and can minimize the chances of malfunction, if the closed-loop system adopts multiple biomarkers. While the reliable methods of sensing bladder events as biomarkers for voiding dysfunction are still in their early stages, the most promising method currently is the direct measurement of bladder pressure or exact detection of bladder events based on analysis of afferent nerve activity. In this paper, we reviewed the current technological status of various closed- loop neuromodulation systems. The treatment for voiding dysfunction using neuromodulation is promising, if biomarkers of bladder events can be accurately detected and controlled through the closed-loop systems.

\section{AUTHOR CONTRIBUTION STATEMENT}

- Full access to all the data in the study and takes responsibility for the integrity of the data and the accuracy of the data analysis: $E P, J W L, B H C, K S L$

- Study concept and design: $E P, M K, K C, B H C, K S L$

- Acquisition of data: $E P, M K, K C, B H C, K S L$

- Analysis and interpretation of data: $E P, M K, K C, B H C, K S L$

- Drafting of the manuscript: EP, JWL

- Critical revision of the manuscript for important intellectual content: $E P, M K, K C, B H C, K S L$

- Statistical analysis: $E P, M K, K C, B H C, K S L$

- Obtained funding: $E P, K S L$

- Administrative, technical, or material support: $E P, B H C, K S L$

-Study supervision: $E P, B H C, K S L$

\section{REFERENCES}

1. Chu CM, Harvie HS, Smith AL, Arya LA, Andy UU. The impact of treatment of overactive bladder on physical activity limitations. J Womens Health (Larchmt) 2016;25:801-5.

2. Hsieh PF, Chiu HC, Chen KC, Chang CH, Chou EC. Botulinum toxin A for the treatment of overactive bladder. Toxins (Basel) 2016 Feb 29;8(3). pii: E59. https://doi.org/10.3390/toxins8030059.

3. Jang HJ, Kwon MJ, Cho KO. Central regulation of micturition and its association with epilepsy. Int Neurourol J 2018;22:2-8.

4. Wolz-Beck M, Reisenauer C, Kolenic GE, Hahn S, Brucker SY, Huebner M. Physiotherapy and behavior therapy for the treatment of overactive bladder syndrome: a prospective cohort study. Arch Gynecol Obstet 2017;295:1211-7.

5. Andersson KE, Birder L. Current pharmacologic approaches in painful bladder research: an update. Int Neurourol J 2017;21:23542.

6. Ladi-Seyedian SS, Nabavizadeh B, Sharifi-Rad L, Kajbafzadeh AM. Pharmacological treatments available for the management of underactive bladder in neurological conditions. Expert Rev Clin Pharmacol 2018;11:193-204.

7. Abello A, Das AK. Electrical neuromodulation in the management of lower urinary tract dysfunction: evidence, experience and future prospects. Ther Adv Urol 2018;10:165-73. 
8. Chai TC, Kudze T. New therapeutic directions to treat underactive bladder. Investig Clin Urol 2017;58(Suppl 2):S99-106.

9. Lay AH, Das AK. The role of neuromodulation in patients with neurogenic overactive bladder. Curr Urol Rep 2012;13:343-7.

10. Sanford MT, Suskind AM. Neuromodulation in neurogenic bladder. Transl Androl Urol 2016;5:117-26.

11. Tanagho EA, Schmidt RA, Orvis BR. Neural stimulation for control of voiding dysfunction: a preliminary report in 22 patients with serious neuropathic voiding disorders. J Urol 1989;142(2 Pt 1):3405.

12. Medtronic, Inc.; premarket approval of the Interstim Sacral Nerve Stimulation (SNS) System--FDA. Notice. Fed Regist 1998;63:4457.

13. Chancellor MB, Chartier-Kastler EJ. Principles of sacral nerve stimulation (SNS) for the treatment of bladder and urethral sphincter dysfunctions. Neuromodulation 2000;3:16-26.

14. McGuire E, Morrissey S, Zhang S, Horwinski E. Control of reflex detrusor activity in normal and spinal injured non-human primates. J Urol 1983;129:197-9.

15. Govier FE, Litwiller S, Nitti V, Kreder KJ Jr, Rosenblatt P. Percutaneous afferent neuromodulation for the refractory overactive bladder: results of a multicenter study. J Urol 2001;165:1193-8.

16. Karam R, Bhunia S, Majerus S, Brose SW, Damaser MS, Bourbeau D. Real-time, autonomous bladder event classification and closedloop control from single-channel pressure data. Conf Proc IEEE Eng Med Biol Soc 2016;2016:5789-92.

17. Lee JW, Kim D, Yoo S, Lee H, Lee GH, Nam Y. Emerging neural stimulation technologies for bladder dysfunctions. Int Neurourol J 2015;19:3-11.

18. Wenzel BJ, Boggs JW, Gustafson KJ, Grill WM. Closed loop electrical control of urinary continence. J Urol 2006;175:1559-63.

19. Sagawa K. Closed-loop physiological control of the heart. Ann Biomed Eng 1980;8:415-29.

20. Paul V, Garratt C, Ward DE, Camm AJ. Closed loop control of rate adaptive pacing: clinical assessment of a system analyzing the ventricular depolarization gradient. Pacing Clin Electrophysiol 1989; 12:1896-902.

21. Lillehei CW, Gott VL, Hodges PC Jr, Long DM, Bakken EE. Transitor pacemaker for treatment of complete atrioventricular dissociation. J Am Med Assoc 1960;172:2006-10.

22. Sutton R, Fisher JD, Linde C, Benditt DG. History of electrical therapy for the heart. Eur Heart J Suppl 2007;9:I3-10.

23. Rickards AF, Norman J. Relation between QT interval and heart rate. New design of physiologically adaptive cardiac pacemaker. $\mathrm{Br}$ Heart J 1981;45:56-61.

24. Miller JD, Yousuf O, Berger RD. The implantable cardioverter-defi- brillator: an update. Trends Cardiovasc Med 2015;25:606-11.

25. Shirvalkar P, Veuthey TL, Dawes HE, Chang EF. Closed-loop deep brain stimulation for refractory chronic pain. Front Comput Neurosci 2018;12:18.

26. Bina RW, Langevin JP. Closed loop deep brain stimulation for PTSD, addiction, and disorders of affective facial interpretation: review and discussion of potential biomarkers and stimulation paradigms. Front Neurosci 2018;12:300.

27. Hoang KB, Cassar IR, Grill WM, Turner DA. Biomarkers and stimulation algorithms for adaptive brain stimulation. Front Neurosci 2017;11:564.

28. Meidahl AC, Tinkhauser G, Herz DM, Cagnan H, Debarros J, Brown P. Adaptive deep brain stimulation for movement disorders: the long road to clinical therapy. Mov Disord 2017;32:810-9.

29. Johnson LA, Nebeck SD, Muralidharan A, Johnson MD, Baker KB, Vitek JL. Closed-Loop deep brain stimulation effects on Parkinsonian motor symptoms in a non-human primate - Is beta enough? Brain Stimul 2016;9:892-6.

30. Powell CR. Conditional electrical stimulation in animal and human models for neurogenic bladder: working toward a neuroprosthesis. Curr Bladder Dysfunct Rep 2016;11:379-85.

31. van Balken MR, Vergunst $H$, Bemelmans BL. The use of electrical devices for the treatment of bladder dysfunction: a review of methods. J Urol 2004;172:846-51.

32. Gaziev G, Topazio L, Iacovelli V, Asimakopoulos A, Di Santo A, De Nunzio C, et al. Percutaneous tibial nerve stimulation (PTNS) efficacy in the treatment of lower urinary tract dysfunctions: a systematic review. BMC Urol 2013;13:61.

33. Gobbi C, Digesu GA, Khullar V, El Neil S, Caccia G, Zecca C. Percutaneous posterior tibial nerve stimulation as an effective treatment of refractory lower urinary tract symptoms in patients with multiple sclerosis: preliminary data from a multicentre, prospective, open label trial. Mult Scler 2011;17:1514-9.

34. de Wall LL, Heesakkers JP. Effectiveness of percutaneous tibial nerve stimulation in the treatment of overactive bladder syndrome. Res Rep Urol 2017;9:145-57.

35. Scaldazza CV, Morosetti C, Giampieretti R, Lorenzetti R, Baroni M. Percutaneous tibial nerve stimulation versus electrical stimulation with pelvic floor muscle training for overactive bladder syndrome in women: results of a randomized controlled study. Int Braz J Urol 2017;43:121-6.

36. de Sèze M, Raibaut P, Gallien P, Even-Schneider A, Denys P, Bonniaud $\mathrm{V}$, et al. Transcutaneous posterior tibial nerve stimulation for treatment of the overactive bladder syndrome in multiple sclerosis: results of a multicenter prospective study. Neurourol Urodyn 2011; 
30:306-11.

37. Burton C, Sajja A, Latthe PM. Effectiveness of percutaneous posterior tibial nerve stimulation for overactive bladder: a systematic review and meta-analysis. Neurourol Urodyn 2012;31:1206-16.

38. Peters KM, Carrico DJ, Perez-Marrero RA, Khan AU, Wooldridge LS, Davis GL, et al. Randomized trial of percutaneous tibial nerve stimulation versus Sham efficacy in the treatment of overactive bladder syndrome: results from the SUmiT trial. J Urol 2010;183:1438-43.

39. Wenzel BJ, Boggs JW, Gustafson KJ, Creasey GH, Grill WM. Detection of neurogenic detrusor contractions from the activity of the external anal sphincter in cat and human. Neurourol Urodyn 2006; 25:140-7.

40. Horvath EE, Yoo PB, Amundsen CL, Webster GD, Grill WM. Conditional and continuous electrical stimulation increase cystometric capacity in persons with spinal cord injury. Neurourol Urodyn 2010;29:401-7.

41. Mendez A, Sawan M, Minagawa T, Wyndaele JJ. Estimation of bladder volume from afferent neural activity. IEEE Trans Neural Syst Rehabil Eng 2013;21:704-15.

42. Wenzel BJ, Boggs JW, Gustafson KJ, Grill WM. Detecting the onset of hyper-reflexive bladder contractions from the electrical activity of the pudendal nerve. IEEE Trans Neural Syst Rehabil Eng 2005; 13:428-35.

43. Macnab AJ. The evolution of near infrared spectroscopy in urology. Biomed Spectrosc Imaging 2014;3:311-44.

44. Macnab A, Shadgan B, Stothers L. Validation of transcutaneous NIRS monitoring of bladder hemodynamics and oxygenation using a rabbit model. Biomed Spectrosc Imaging 2013;2:91-9.

45. Macnab A, Friedman B, Shadgan B, Stothers L. Bladder anatomy physiology and pathophysiology: elements that suit near infrared spectroscopic evaluation of voiding dysfunction. Biomed Spectrosc Imaging 2012;1:223-35.

46. Macnab AJ, Stothers L, Shadgan B. Wireless near-infrared spectroscopy (NIRS) of the bladder detrusor during uroflow in ambulant children and adults. J Urology 2009;181(4 Suppl):600-1.

47. Macnab AJ, Shadgan B, Stothers L, Afshar K. Ambulant monitoring of bladder oxygenation and hemodynamics using wireless near-infrared spectroscopy. Can Urol Assoc J 2013;7:E98-104.

48. Macnab A, Shadgan B, Afshar K, Stothers L. Near-infrared spectroscopy of the bladder: new parameters for evaluating voiding dysfunction. Int J Spectrosc 2011;2011:Article ID 814179.

49. Fong D, Alcantar AV, Gupta P, Kurzrock E, Ghiasi S. Non-invasive bladder volume sensing for neurogenic bladder dysfunction management. In: 2018 IEEE 15th International Conference Wearable and Implantable Body Sensor Networks (BSN): 2018 Mar 4-7; Las Vegas, USA. Piscataway (NJ): Institute of Electrical and Electronics Engineers; 2018. p. 82-5.

50. Molavi B, Shadgan B, Macnab AJ, Dumont GA. Noninvasive optical monitoring of bladder filling to capacity using a wireless near infrared spectroscopy device. IEEE Trans Biomed Circuits Syst 2014;8:325-33.

51. Clausen I, Tvedt LG, Glott T. Measurement of urinary bladder pressure: a comparison of methods. Sensors (Basel) 2018 Jul 3;18(7). pii: E2128. https://doi.org/10.3390/s18072128.

52. Dakurah MN, Koo C, Choi W, Joung YH. Implantable bladder sensors: a methodological review. Int Neurourol J 2015;19:133-41.

53. Opisso E, Borau A, Rijkhoff NJ. Urethral sphincter EMG-controlled dorsal penile/clitoral nerve stimulation to treat neurogenic detrusor overactivity. J Neural Eng 2011;8:036001.

54. Bruns TM, Gaunt RA, Weber DJ. Multielectrode array recordings of bladder and perineal primary afferent activity from the sacral dorsal root ganglia. J Neural Eng 2011;8:056010.

55. Majerus SJ, Fletter PC, Ferry EK, Zhu H, Gustafson KJ, Damaser MS. Suburothelial bladder contraction detection with implanted pressure sensor. PLoS One 2017;12:e0168375.

56. Rutter EM, Langdale CL, Hokanson JA, Hamilton F, Tran H, Grill $\mathrm{WM}$, et al. Detection of bladder contractions from the activity of the external urethral sphincter in rats using sparse regression. IEEE Trans Neural Syst Rehabil Eng 2018;26:1636-44.

57. Ross SE, Ouyang Z, Rajagopalan S, Bruns TM. Evaluation of decoding algorithms for estimating bladder pressure from dorsal root ganglia neural recordings. Ann Biomed Eng 2018;46:233-46.

58. Brindley GS, Donaldson PE. Electrolytic current-control elements for surgically implanted electrical devices. Med Biol Eng Comput 1986;24:439-41.

59. Kim A, Powell CR, Ziaie B. An Universal packaging technique for low-drift implantable pressure sensors. Biomed Microdevices 2016; $18: 32$.

60. Young DJ, Cong P, Suster MA, Damaser M. Implantable wireless battery recharging system for bladder pressure chronic monitoring. Lab Chip 2015;15:4338-47.

61. Khurram A, Ross SE, Sperry ZJ, Ouyang A, Stephan C, Jiman AA, et al. Chronic monitoring of lower urinary tract activity via a sacral dorsal root ganglia interface. J Neural Eng 2017;14:036027.

62. Kim MK, Kim H, Jung YS, Adem KMA, Bawazir SS, Stefanini C, et al. Implantable bladder volume sensor based on resistor ladder network composed of conductive hydrogel composite. Conf Proc IEEE Eng Med Biol Soc 2017;2017:1732-5. 